\title{
An exploration of the association between vitamin $D$ intake in early pregnancy and fetal and maternal clinical outcomes
}

\author{
M. Hayden ${ }^{1}$, S. Cawley ${ }^{1}$, M.J. Turner ${ }^{2}$ and D. McCartney ${ }^{1}$ \\ ${ }^{1}$ School of Biological Sciences and Environmental Sustainability and Health Institute (ESHI), Dublin Institute of \\ Technology, Dublin 8, Republic of Ireland and ${ }^{2} U C D$ Centre for Human Reproduction, \\ Coombe Women and Infants University Hospital, Dublin 8, Republic of Ireland
}

Evidence indicates that sub-optimal vitamin D status $(25(\mathrm{OH}) \mathrm{D}<50 \mathrm{nmol} / 1)$ is common amongst pregnant women in Ireland ${ }^{(1-3)}$, with mean dietary intakes estimated to be between 1.9-3.6 $\mathrm{g} /$ day $^{(1,4)}$. This is of a particular concern, as the fetus relies on maternal intakes and stores of vitamin D to meet its demands ${ }^{(5)}$. During pregnancy, vitamin D influences calcium and phosphorous homeostasis ${ }^{(6)}$ to enable fetal bone mineral accretion. More recent evidence however, suggests that maternal serum $25(\mathrm{OH}) \mathrm{D}$ levels may also influence extra-skeletal maternal and fetal health outcomes; including birthweight and the risk of pre-eclampsia (PET), gestational diabetes mellitus (GDM), pre-term birth and small for gestational age (SGA) ${ }^{(6,7)}$.

This observational study examined maternal vitamin D intakes in the first trimester. Women were recruited at their convenience after sonographic confirmation of a singleton pregnancy in the first trimester. Maternal body composition was measured using 8-electrode bioelectrical impedance analysis, and dietary data collected using an interviewer-assisted 4-day retrospective diet history (DH) in combination with a food frequency questionnaire (FFQ). All reported portion sizes were fully quantified using food portion size estimation tools. Nutrient analysis was carried out using Nutritics version 3.7 University Edition which included the most up-to-date nutrient composition for fortified foods.

Among the 720 women recruited, dietary intake data was available for the full sample $(n=720)$ and supplement data for the majority $(n=634)$. Of the full sample, 238 were classified as energy under-reporters. Amongst the plausible dietary reporters $(n=482 / 720)$, median vitamin D intake from diet alone was $2.7 \mu \mathrm{g} /$ day, with $2.7 \%$ achieving the EAR (10 $\mu \mathrm{g} /$ day) ${ }^{(8)}$ from food only. Median intake from diet and supplements combined was $6.3 \mu \mathrm{g}$ /day, with $48.2 \%$ of supplement users achieving the EAR. Vitamin D supplement usage for the entire sample was $61.2 \%$. No association was reported between achievement of the EAR and fetal or maternal health outcomes. When multivariate analyses were performed, those experiencing relative income poverty were less likely to supplement, while planning a pregnancy persisted as a positive predictor of vitamin D supplementation.

This study suggests that dietary vitamin D intakes are sub-optimal in pregnancy, and that supplements make a critical contribution to overall intakes. However, it also indicates that supplementation is less common amongst low SES women, particularly those who don't plan their pregnancy. The study has implications for public health interventions in relation to vitamin D in pregnancy, and in relation to future food fortification policy.

1. O'Riordan M, Kiely M, Higgins J, Cashman K. (2008) Ir Med J 101(8), 240-242.

2. Holmes VA Barnes MS, Alexander HD, et al. (2009) Br J Nutr 102(06), 876-881.

3. Institute of Obstetricians and Gynaecologists, Royal College of Physicians of Ireland and Directorate of Clinical Strategy and Programmes, Health Service Executive Clinical Practise Guideline Nutrition for Pregnancy. Version 1.1, 27th edition, 2013.

4. McGowan C Byrne J, Walsh J, et al. (2011) Eur J Clin Nutr 65(9), 1076-1078.

5. Langley-Evans SC. (2006) Pro Nut Soc 65(01), 97-105.

6. Wei SQ, Qi HP, Luo ZC, Fraser WD. (2013) J Matern Fetal Neonatal Med 26(9), 889-899.

7. Bärebring L, Bullarbo M, Glantz A, et al. (2016) PLoS ONE 11(3), e 0152198.

8. Cashman K, Kiely M.? (2014) J Hum Nutr Diet 27(5), 434-442. 